Case report

\title{
Strangulated Morgagni hernia and sliding hiatal hernia: A rare association.
}

\author{
Ferjaoui Wael 1,2* Karouia Souhaiel1,2, Talbi Ghofrane1,2, Gabsi Soufien ${ }^{1,2}$, Bayar \\ Rached ${ }^{1,2}$, Khalfallah Mohamed Tahar ${ }^{1,2}$.
}

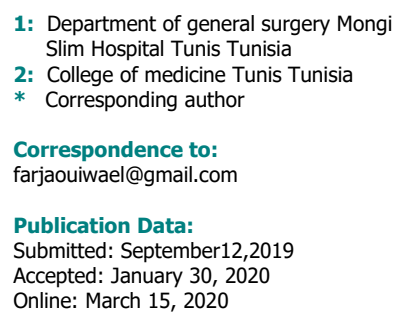

This article was subject to full peer-review.

This is an open access article distributed under the terms of the Creative Commons Attribution NonCommercial License 4.0 (CCBY-NC) allowing sharing and adapting.

Share: copy and redistribute the material in any medium or format.

Adapt: remix, transform, and build upon the licensed material.

the work provided must be properly cited and cannot be used for commercial purpose.

\section{Abstract}

Morgagni hernias are congenital diaphragmatic hernias accounting for 3 to $5 \%$ of all diaphragmatic hernias. The association with hiatal hernia is rarely reported in the literature. We report another case of a right Morgagni hernia revealed by an acute intestinal obstruction associated with a sliding hernia hiatal.

The aim is to discusses the clinical presentation and to highlight the management characteristics.

\section{Keywords:}

Morgagni Hernia; Hiatal Hernia Type I; intestinal obstruction; surgery.

\section{Introduction}

The diaphragm muscle has some weak spots giving rise to hernias. Morgagni type is the organ herniation through the para-retrosternal hiatus. It represents $3-5 \%$ of all diaphragmatic Hernias and occurs mostly on the right side [1]. Hiatal hernias with the protrusion of a portion of the stomach into the thoracic cavity are the most common. The association of these hernias in the same patient remains exceptional. Only a few cases have been described in the literature [2].

\section{Observation}

A 75-year-old female patient with no pertinent past medical history presented to our emergency department with acute abdominal pain and recurrent episodes of vomiting. On physical examination, she was apyrexial and vitally stable. Abdomen was distended and tender. Breath sounds were decreased on the right side of the chest. The abdomen Xray erect showed multiple differential air-fluid levels. The chest radiography showed an air-fluid level in the right side of mediastinum (figure 1). A computed tomogram (CT) of the chest and the abdomen revealed a Morgagni hernia through a retrosternal right diaphragmatic defect with protrusion of the transverse colon and a part of the great omentum. A sliding hiatal hernia was simultaneously discovered with visualized partially protruded stomach in the thoracic cavity (figure 2). 


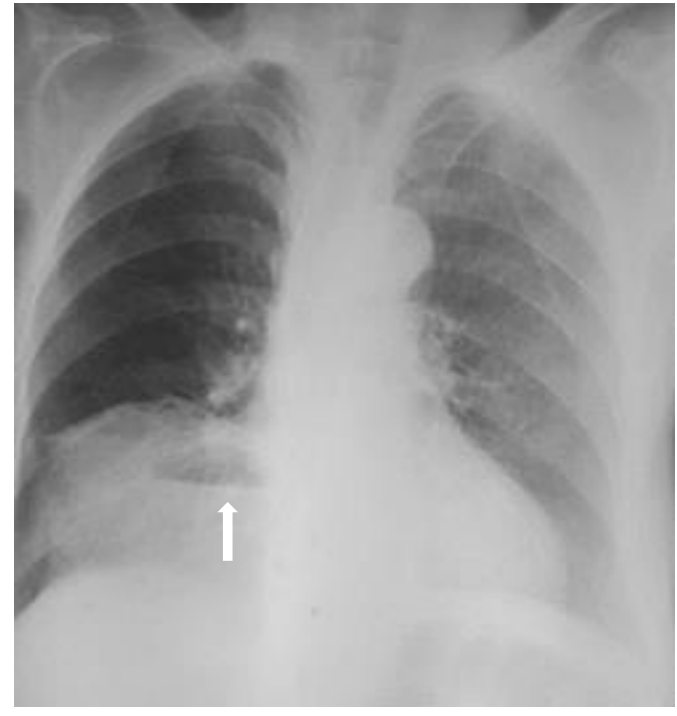

Figure 1: Chest x-ray revealing a mediastinal air-fluid level

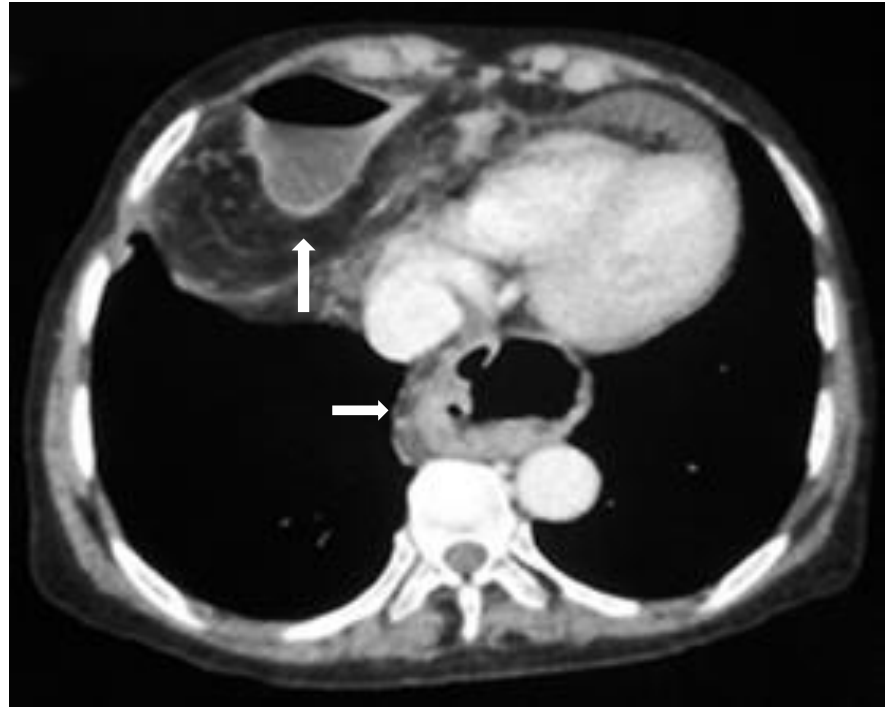

Figure 2: Computed tomography showing coexistence of both hernias

The patient underwent midline laparotomy. Intraoperative findings were in favor of a defect in the diaphragm measuring $5 * 4 \mathrm{~cm}$. This defect is located in the right side of the retrosternal region. The hernia contained the transverse colon and omentum with stricture effect causing bowel obstruction and dilatation. The adhesions were released. The contents were reduced into the abdomen. The diaphragmatic defect was repaired using non resorbable sutures (figure 3). The repair of the hiatal hernia was postponed for further endoscopic investigations. The postoperative courses were uneventful. The post-operative chest $\mathrm{x}$-ray was unremarkable. The patient was discharged on the 4th day after surgery.

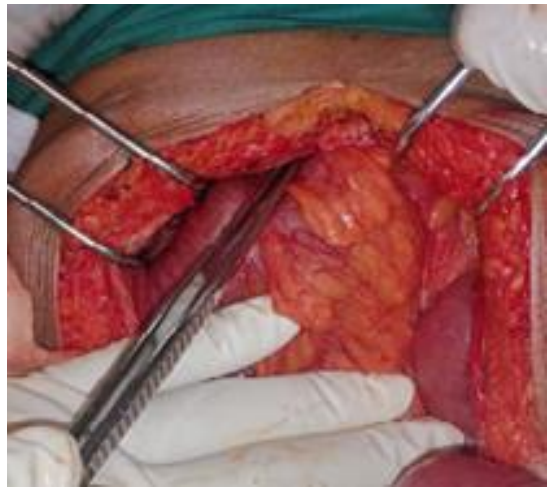

A

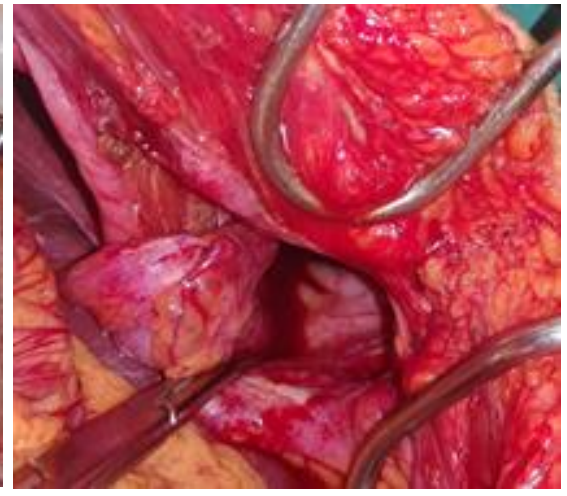

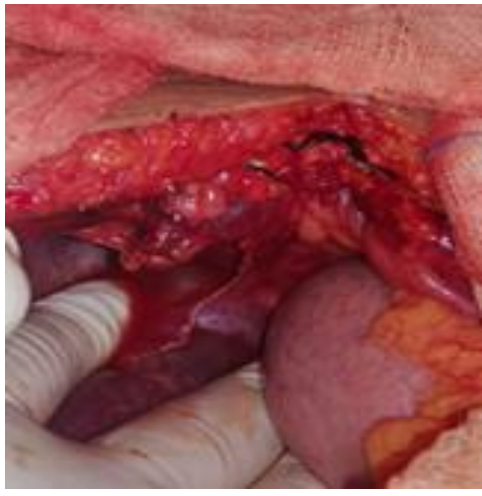

C

Figure 3

A: Intraoperative features of diaphragmatic hernia with herniated omentum and transverse colon.

B: Intraoperative features of Morgagni's hernial defect.

C: repair of the defect using non resorbable sutures 


\section{Discussion}

The coexistence of two non-traumatic diaphragmatic hernias is an extremely rare clinical condition. Only few cases have been previously reported since 1958 . Morgagni hernia is a congenital diaphragmatic hernia that occurs through the parasternal hiatus of the diaphragm. It is more often right sided $(90 \%)$ but it can be left sided (2\%) or even bilateral $(8 \%)[3,4]$. Morgagni hernia is usually asymptomatic and has insidious evolution. Otherwise presenting symptoms may include chest or abdominal pain, dyspnea, dysphagia, or respiratory distress. The diagnosis is made incidentally or at the moment of complications in more than $30 \%$ of cases [5]. Our patient developed intestinal obstruction due to colon incarceration. The chest and abdomen computed tomography is the is more accurate for the diagnosis specially for asymptomatic patients [6]. The sliding hiatal hernia (type I) is a non-traumatic acquired hernia characterized by protrusion of the gastroesophageal junction into the posterior mediastinum through the esophageal hiatus. The association of these two hernias is uncommon and usually reported in adult patients [7]. The presence of a diaphragmatic hernia reduces the intra-abdominal pressure and might decrease the likelihood of a second diaphragmatic hernia formation. Laparoscopic repair is the best surgical approach for all diaphragmatic hernias. The non-resorbable sutures is still widely performed in case of small defects. The use of laparoscopic mesh has made easier the repair of big hernias. The laparoscopic fundoplication is still the gold standard of the treatment of hiatal hernias. The laparoscopic access allows the repair of both hernias without added morbidity. However, In the open technique the procedure should be reasonably discussed specially in elderly patients. Due to the risk of complications, most of the authors have recommended simultaneous repair for the two hernias even in asymptomatic patients [8].

\section{Conclusions}

Association between Morgagni and hiatal hernias type I is exceptional. Computed tomography (CT) of the chest and the abdomen is important for the diagnosis. Surgical treatment should be applied in Morgagni hernia complicated with strangulation. On the other hand, simultaneous treatment of hiatal hernia type I is nonconsensual.

\section{Conflict of Interest: None}

\section{References}

[1] Modi M, Dey AK, Mate A, Rege S. Strangulated Morgagni's Hernia: A Rare Diagnosis and Management. Case Rep Surg. 2016; 2016:2621383. [2] Hassan A, Alabdrabalmeer M, Alealiwi M, Danan OA, Alshomimi S. Incidental Morgagni hernia found during laparoscopic repair of hiatal hernia: Case report \& review of literature. Int J Surg Case Rep. 2019; 57:97-101.

[3] Zhou Z-L, Li H, Li J-F, Liu Y, Wang C, Wang J. A Rare Case of Laparoscopic Repair of Simultaneously Occurring Morgagni and Paraesophageal Hernias. Ann Thorac Cardiovasc Surg. 2016; 22:112-5.

[4] Nasr A, Fecteau A. Foramen of Morgagni Hernia: Presentation and Treatment. Thorac Surg Clin. 2009; 19:463-8.

[5] Lee SY, Kwon JN, Kim YS, Kim KY. Strangulated Morgagni hernia in an adult: Synchronous prolapse of the liver and transverse colon. Ulus Travma Acil Cerrahi Derg. 2018; 24:376-8.

[6] Chaturvedi A, Rajiah P, Croake A, Saboo S, Chaturvedi A. Imaging of thoracic hernias: types and complications. Insights Imaging. 2018;9(6):9891005.

[7] Fu S, Carton M, Ghaderi I, Galvani C. Robotic-assisted simultaneous repair of paraesophageal hernia and Morgagni hernia: technical report. J Laparoendosc Adv Surg Tech 2018; 28:745-50.

[8] Patel AP, Samsami D, Lee C, Lakha A, Ehrenpreis ED. The waiting game: laparoscopic repair of dual Morgagni and paraesophageal hernias in an elderly, infirm patient. J Surg Case Rep. 2019; 8: rjz225. 